\title{
Could semiquantitative analysis of real-time ultrasound elastography distinguish more liver parenchyma alterations of nonalcoholic fatty liver disease in patients with polycystic ovary syndrome?
}

$\mathrm{Na} \mathrm{Di}^{1,2}$, Xinchuan Zhou' ${ }^{1}$ Yaxiao Chen², Xiaomiao Zhao ${ }^{2}$, Lin $\mathrm{Li}^{2}$, Linlin Jiang' ${ }^{2}$ Baoming Luo', Xiaoli Chen², Dongzi Yang ${ }^{2}$

${ }^{1}$ Department of Ultrasound, Sun Yat-sen Memorial Hospital of Sun Yat-sen University, Guangzhou, China

${ }^{2}$ Department of Obstetrics and Gynecology, Sun Yat-sen Memorial Hospital of Sun Yat-sen University, Guangzhou, China

Correspondence to: Dongzi Yang

Department of Obstetrics and Gynecology,

Sun Yat-sen Memorial Hospita of Sun Yat-sen University West Yanjiang Road 107, Guangzhou, China

yangdz@mail.sysu.edu.cn

Received on Oct/24/2018 Accepted on Dec/12/2018

DOI: 10.20945/2359-3997000000119

\begin{abstract}
Objective: Nonalcoholic fatty liver disease is the commonest diffuse liver disease, of which women with polycystic ovary syndrome are at an increased risk. The aim of the present study was to assess the diagnostic value of the semiquantitative strain parameters of real-time ultrasound elastography for nonalcoholic fatty liver disease in patients with polycystic ovary syndrome. Subjects and methods: Thirty-five polycystic ovary syndrome patients with nonalcoholic fatty liver disease, 70 polycystic ovary syndrome patients without nonalcoholic fatty liver disease, and 70 healthy female controls of reproductive age were included. All participants underwent ultrasonic examination and semiquantitative analysis of real-time ultrasound elastography of the liver. Results: Main semi quantitative strain parameters, such as average strain value, differed significantly among groups polycystic ovary syndrome with nonalcoholic fatty liver disease, polycystic ovary syndrome without nonalcoholic fatty liver disease, and control ( $87.02 \pm 10.16$ vs. $96.31 \pm 11.44$ vs. $104.49 \pm 7.28, p<0.001)$. Clinical and laboratory parameters differed significantly between the two subgroups with low or high average strain value. For diagnostic value of average strain value for elevated aminotransferase, the area under the curve was 0.808 (range 0.721-0.895). In multiple linear regression analysis, polycystic ovary syndrome, waist circumference, and metabolic syndrome were stand-alone independent factors associated with average strain value among subjects without nonalcoholic fatty liver disease. Conclusion: Semiquantitative real-time ultrasound elastography analysis could distinguish liver parenchyma alterations in patients with polycystic ovary syndrome more sensitively. The diagnostic value of the proposed method for nonalcoholic fatty liver disease need further research. Arch Endocrinol Metab. 2019;63(2):128-36
\end{abstract}

Keywords

Polycystic ovary syndrome; nonalcoholic fatty liver disease; real-time ultrasound elastography; semiquantitative analysis; metabolic disturbances

\section{INTRODUCTION}

$\mathrm{N}$ onalcoholic fatty liver disease (NAFLD) is the commonest diffuse liver disease, comprising the spectrum of liver damage from simple hepatic steatosis to nonalcoholic steatohepatitis to advanced fibrosis and cirrhosis, in patients without significant alcohol consumption and any other cause of liver diseases. The worldwide prevalence of NAFLD, which is $5 \%-58 \%$ in the general population, has increased over time and has become a major public health problem (1). In the Chinese population from Hong Kong, the prevalence of NAFLD is as high as $42 \%$ according to a 2015 report (2). NAFLD is also used as a marker of severe metabolic disorders.

Polycystic ovary syndrome (PCOS) is the most common endocrine disorder among women of reproductive age, and the prevalence varies from $5 \%$ to $20 \%(1,3)$. It is also a metabolic disease according to high prevalence of the accompanying metabolic disturbances, which may pose a high long-term risk of cardiovascular diseases for patients with PCOS (4). Women with PCOS are at an increased risk of NAFLD as reported previously: the prevalence of NAFLD varies from $23.8 \%$ to $86.79 \%$ among women with PCOS 
$(5,6)$. Accordingly, it has been suggested that women with PCOS should be screened for liver disease more actively (7).

Although a liver biopsy is still considered the gold standard for detection of a liver disease such as fibrosis, this procedure is invasive and is associated with possible problems such as bleeding and severe pain, sampling errors, and interobserver variability (8). Computed tomography (CT) or magnetic resonance imaging (MRI) can quantify liver fat, but CT involves ionizing radiation, and both methods are costly (9). Conventional ultrasonography has been used for detection of NAFLD in daily practice, but its accuracy is limited by subcutaneous fat thickness of patients and the operator's experience. Furthermore, it cannot provide information about mechanical properties of the tissue. It is impractical to use these methods for assessment and frequent monitoring of NAFLD in PCOS patients of reproductive age. Real-time tissue elastography (RTE), one of the ultrasound elastography technologies, has been rapidly evolving in the last decade, being used for diagnosis of focal lesions in various organs. According to recent reports, RTE is also useful for assessment of fibrosis in patients with chronic liver diseases $(10,11)$. With the improvements in RTE, semiquantitative analysis of strain was developed. Parameters including the average strain value (MEAN) of RTE are reported to be useful for early diagnosis and staging of liver fibrosis (12-18).

On the other hand, application of RTE with semiquantitative parameters to NAFLD is rarely reported, never in PCOS women of reproductive age. The aims of our study were to analyze semiquantitative strain parameters of RTE of the liver in PCOS patients with or without NAFLD as compared to healthy controls and then to evaluate the diagnostic value of these parameters for NAFLD in patients with PCOS.

\section{SUBJECTS AND METHODS}

\section{Sample size estimate}

No previous study had reported the efficacy of RTE in diagnose of NAFLD in patients with PCOS. According to report about RTE for assessment of liver fibrosis in chronic hepatitis B (18), a sample size of 5 in each group has a power of 0.949 at $5 \%$ significance to detect a difference of 6.39 in the value of MEAN between cases and controls calculated by PASS 11(NCSS LLC,
USA). We recruited more than 5 participants for each group. A total of 35 PCOS patients with NAFLD, 70 PCOS patients without NAFLD, and 70 healthy female controls aged 20 to 40 years were recruited in our Hospital from May 27, 2015, to November 31, 2015.

\section{Patients and controls}

The study was approved by the Institutional Review Board of our Hospital. All participants provided written informed consent. The Chinese Clinical Trial Registry (http://www.chictr.org/en/) number is ChiCTROOC-15006452. Detailed data on all participates were deposited in the Research Electronic Data Capture (REDCap) databases.

PCOS was diagnosed according to the Rotterdam 2003 criteria (19), two of the following three criteria had to be met: 1) oligomenorrhea and/or anovulation, 2) clinical and/or biochemical hyperandrogenism, 3 ) polycystic ovaries (PCO) (presence in each ovary of 12 or more follicles measuring 2 to $9 \mathrm{~mm}$ in diameter and/or increased ovarian volume: more than $10 \mathrm{~mL}$ ). NAFLD was diagnosed according to conventional criteria (conventional ultrasonography) (20). Controls were defined as healthy non pregnant, amenorrheic women without endocrine disorders, $\mathrm{PCO}$, and NAFLD.

Women were excluded from the study if they had a medical history that included other known liver diseases, ovarian surgery, a malignant tumor, tobacco smoking, and alcohol drinking, other endocrine diseases, or a severe cardiocerebrovascular disease or if they were taking medication that may affect metabolism, blood pressure, or liver function during the last 3 months.

\section{Bioclinical tests}

The medical history including menstrual regularity, medications, and past diseases was recorded. All anthropometric data were measured and recorded including waist circumference (WC), hip circumference (HC), waistline/hipline ratio (WHR), body mass index (BMI), acne score, modified Ferriman-Gallwey score ( $\mathrm{mFG}$ ) for body hair evaluation, systolic blood pressure (SBP), and diastolic blood pressure (DBP).

Basal follicle-stimulating hormone (FSH), luteinizing hormone (LH), prolactin (PRL), estradiol (E2), and total testosterone (TT) were quantified by chemiluminesce Immunoassay on an automatic biochemistry analyzer (DXI800, Beckman COULTER, Inc. USA) during the early follicular 
phase or first 3 days of progestin withdrawal bleeding. Anti-Müllerian hormone $(\mathrm{AMH})$ and sex hormonebinding globulin (SHBG) were analyzed by an enzyme-linked immunosorbent assay (ELISA) on a ELx808 plate reader (Biotek Instruments, Inc. USA). Serum transaminases, plasma glucose, insulin, and lipid parameters were measured using standard methods on an automatic chemiluminescence immunoassay system (ADVIA Centaur xp, SIEMENS, Germen).

Oligomenorrhea/amenorrhea and PCO were defined according to the revised Rotterdam criteria. Biochemical hyperandrogenism (bHA) was defined as a total testosterone level of $2.39 \mathrm{nM}$ or greater (21). Hirsutism was defined as $\mathrm{mFG} \geq 5$ as previously reported (22). The acne score was measured according to a previous report (23). Homeostatic model assessment for insulin resistance (HOMA-IR) was calculated as fasting blood glucose $(\mathrm{FBG}) \times$ fasting insulin $(\mathrm{FIN}) \div 22.5$. Insulin resistance (IR) was defined as HOMA-IR $\geq 2.14$ and FIN $\geq 12.6 \mathrm{mIU} / \mathrm{L}(24,25)$. Impaired fasting glucose tolerance (FGT), impaired glucose tolerance (IGT) and type 2 diabetes mellitus (DM) were diagnosed according to 2006 WHO criteria (26). Hyperlipidemia was defined as cholesterol $(\mathrm{CHOL}) \geq 6 \mathrm{mM}$, triglycerides $(\mathrm{TG}) \geq 2.3$ $\mathrm{mM}$, or low-density lipoprotein cholesterol (LDL-C) $\geq 3.6 \mathrm{mM}$. Elevated aminotransferase was defined as alanine transaminase (ALT) or aspartate transaminase $($ AST) $\geq 40 \mathrm{U} / \mathrm{L}$. Central obesity was defined as the waistline $\geq 80 \mathrm{~cm}$. Elevated blood pressure (EBP) was defined as $\mathrm{SBP} \geq 130 \mathrm{mmHg}$ or $\mathrm{DBP} \geq 85 \mathrm{mmHg}$. Metabolic syndrome (MS) was diagnosed according to NCEP-ATP III criteria (27).

\section{Ultrasound examination and RTE}

Transvaginal ultrasonography was performed in the early follicular phase if menses were regular or randomly if menses were irregular. Antral follicle count (AFC) was recorded.

All procedures were performed by a single operator with 10 years of experience in transabdominal ultrasonography who had specialized in elastography for the last 5 years. The operator was blinded to the clinical data of all the participants.

The ultrasonic examination (US) was performed by means of HI VISION PREIRUS (Hitachi Medical, Tokyo, Japan), which has both conventional ultrasonography and RTE capabilities. Conventional US was performed with a $3.5 \mathrm{MHz}$ probe. A7-3MHz linear transducer (L52) was used for RTE.
RTE was performed as reported previously (16). The region of interest window was set to $1 \mathrm{~cm}$ under the Glisson's capsule in the right lobe, avoiding bile ducts and bile cysts. The pressure causing liver tissue strain came from subjects' own heartbeat. The RTE software (EZU-TESHl, Hitachi) was used to generate histograms. Three effective acquisitions were performed for each patient. The mean of the total of three measurements served as a representative strain value. We measured 11 characteristic parameters of elastography imaging including MEAN in the range of 0-255 arbitrary units (a.u.) and the area ratio of a lowstrain region (AREA, \%) as the main characteristics of liver stiffness. Standard deviation of the relative strain value (SD) was also determined.

\section{Data analysis}

The results are presented as mean \pm standard deviation or as a rate. The one-sample Kolmogorov-Smirnov test was used to determine whether the distribution of variants was normal. MEANs were compared among the groups using the $t$ test or one-way analysis of variance (ANOVA). When equal variances were not assumed, variants were compared by nonparametric tests (MannWhitney $U$ test or Kruskal-Wallis test). Categorical data were compared among the groups using the chisquared test. Receiver operating characteristic (ROC) curves were generated to analyze the diagnostic value of MEAN for elevated aminotransferase. Multiple linear regression analysis was used to access the correlation between MEAN or AREA and clinical parameters. These analyses were performed in the SPSS software, version 13.0 for Windows (SPSS, Inc., Chicago, IL, USA), and statistical significance was assumed at $p<0.05$.

\section{RESULTS}

BMI, WC, WHR, SBP, DBP, FBG, two-hour glucose (2hGLU), FIN, HOMA-IR, TG, ALT, and AST levels significantly decreased in the following order: group PCOS with NAFLD $>$ group PCOS without NAFLD $>$ control group $(p<0.05)$. HDL-C levels showed an inverse trend among the three groups $(p<0.05)$. LDL-C, apoB, AMH, LH, TT, mFG, menstrual cycles, and AFC were much higher in the PCOS groups than in the control group $(p<0.05)$, without significant difference between the two PCOS groups. Group PCOS without NAFLD had a significantly higher 
level of apoA than did group PCOS with NAFLD and control group $(p<0.05)$ (Table 1$)$.

RTE for livers are shown in Figure 1. The MEAN value was the lowest in group PCOS with NAFLD, intermediate in group PCOS without NAFLD, and highest in the control group $(87.02 \pm 10.16$ vs. 96.31 \pm 11.44 vs. $104.49 \pm 7.28, p<0.001)$. The AREA value was the highest in group PCOS with NAFLD, intermediate in group PCOS without NAFLD, and lowest in the control group $(42.49 \pm 9.41$ vs. 33.58 \pm 10.91 vs. $24.55 \pm 8.14, p<0.001) . S D$ values were significantly different among the three groups in the same descending order as for AREA $(68.78 \pm 5.21$ vs. $64.08 \pm 6.73$ vs. $58.25 \pm 6.58, p<0.001$ ) (Figure 2).

To validate the clinical utility of MEAN, all subjects were subdivided into two subgroups according to MEAN levels. The cutoff value of MEAN $(<91.7)$ was set to the 5 th percentile of the average relative strain value in 70 healthy controls. All 175 subjects were subdivided into group A (MEAN value

Table 1. Baseline clinical characteristics and laboratory parameters of all subjects

\begin{tabular}{|c|c|c|c|c|c|c|}
\hline & $\begin{array}{c}\text { PCOS } \\
\text { With NAFLD }\end{array}$ & $\begin{array}{l}\text { PCOS without } \\
\text { NAFLD }\end{array}$ & Control & $P_{1}$ & $\mathbf{P}_{2}$ & $\mathbf{P}_{3}$ \\
\hline $\mathbf{N}$ & 35 & 70 & 70 & & & \\
\hline $\mathrm{Age}^{\mathrm{a}}$ & $29 \pm 4.41$ & $28.83 \pm 4.51$ & $28.97 \pm 4.71$ & NS & NS & NS \\
\hline $\mathrm{BM}^{\mathrm{a}}\left(\mathrm{kg} / \mathrm{m}^{2}\right)$ & $25.41 \pm 3.40$ & $22.81 \pm 2.67$ & $21.15 \pm 2.61$ & $<0.001$ & $<0.001$ & $<0.001$ \\
\hline$W C^{a}(\mathrm{~cm})$ & $86.23 \pm 7.94$ & $77.80 \pm 8.21$ & $72.91 \pm 8.53$ & $<0.001$ & $<0.001$ & $<0.001$ \\
\hline WHR $^{\mathrm{a}}$ & $0.8805 \pm 0.0528$ & $0.8337 \pm 0.0619$ & $0.8013 \pm 0.0735$ & 0.001 & $<0.001$ & 0.004 \\
\hline $\mathrm{SBPa}(\mathrm{mmHg})$ & $122.68 \pm 12.79$ & $117.18 \pm 10.38$ & $111.01 \pm 9.48$ & 0.021 & $<0.001$ & $<0.001$ \\
\hline $\mathrm{DBPa}^{2}(\mathrm{mmHg})$ & $79.89 \pm 9.51$ & $75.85 \pm 8.96$ & $69.66 \pm 7.14$ & 0.033 & $<0.001$ & $<0.001$ \\
\hline $\mathrm{FBG}^{\mathrm{b}}(\mathrm{mmol} / \mathrm{L})$ & $5.28 \pm 0.83$ & $4.91 \pm 0.45$ & $4.72 \pm 0.31$ & 0.007 & $<0.001$ & 0.012 \\
\hline 2hGLUb & $7.75 \pm 2.39$ & $6.50 \pm 1.94$ & $5.53 \pm 0.93$ & 0.005 & $<0.001$ & $<0.001$ \\
\hline $\mathrm{FIN}^{\mathrm{b}}(\mathrm{mlU} / \mathrm{L})$ & $25.39 \pm 13.95$ & $14.15 \pm 6.68$ & $9.20 \pm 5.51$ & $<0.001$ & $<0.001$ & $<0.001$ \\
\hline HOMA-IR & $6.01 \pm 4.68$ & $3.07 \pm 1.64$ & $1.96 \pm 1.34$ & $<0.001$ & $<0.001$ & $<0.001$ \\
\hline $\mathrm{CHOL}^{\mathrm{b}}(\mathrm{mmol} / \mathrm{L})$ & $5.01 \pm 1.35$ & $4.74 \pm 0.74$ & $4.59 \pm 0.72$ & NS & NS & NS \\
\hline $\mathrm{TG}^{\mathrm{b}}(\mathrm{mmol} / \mathrm{L})$ & $1.92 \pm 1.02$ & $1.25 \pm 0.72$ & $0.81 \pm 0.28$ & $<0.001$ & $<0.001$ & $<0.001$ \\
\hline $\mathrm{HDLC}^{\mathrm{b}}(\mathrm{mmol} / \mathrm{L})$ & $1.21 \pm 0.25$ & $1.53 \pm 0.37$ & $1.44 \pm 0.24$ & $<0.001$ & $<0.001$ & NS \\
\hline $\mathrm{LDLC}^{\mathrm{b}}(\mathrm{mmol} / \mathrm{L})$ & $3.25 \pm 1.08$ & $2.90 \pm 0.65$ & $2.55 \pm 0.67$ & NS & $<0.001$ & 0.002 \\
\hline apoA $(g / L)$ & $1.36 \pm 0.33$ & $1.57 \pm 0.36$ & $1.40 \pm 0.23$ & 0.002 & 0.002 & 0.010 \\
\hline apoB $(g / L)$ & $0.88 \pm 0.24$ & $0.79 \pm 0.21$ & $0.70 \pm 0.12$ & NS & 0.001 & 0.017 \\
\hline $\mathrm{ALT}^{\mathrm{b}}(\mathrm{U} / \mathrm{L})$ & $27.44 \pm 16.25$ & $15.52 \pm 4.50$ & $11.03 \pm 4.89$ & 0.001 & $<0.001$ & $<0.001$ \\
\hline $\mathrm{AST}^{\mathrm{b}}(\mathrm{U} / \mathrm{L})$ & $39 \pm 30.91$ & $14.69 \pm 8.74$ & $15.17 \pm 3.19$ & $<0.001$ & $<0.001$ & 0.030 \\
\hline $\mathrm{AMH}^{\mathrm{b}}(\mathrm{ng} / \mathrm{dL})$ & $9.99 \pm 4.67$ & $9.67 \pm 4.52$ & $4.52 \pm 3.16$ & NS & $<0.001$ & $<0.001$ \\
\hline TTa (nmol/L) & $1.9355 \pm 0.7381$ & $1.80 \pm 1.1471$ & $1.2114 \pm 0.5210$ & NS & $<0.001$ & $<0.001$ \\
\hline $\mathrm{PRL}^{\mathrm{a}}(\mathrm{ng} / \mathrm{mL})$ & $15.11 \pm 7.67$ & $14.53 \pm 7.80$ & $15.09 \pm 6.81$ & NS & NS & NS \\
\hline $\mathrm{FSH}^{\mathrm{a}}(\mathrm{mlU} / / \mathrm{mL})$ & $6.89 \pm 1.87$ & $6.50 \pm 2.11$ & $7.84 \pm 2.55$ & NS & NS & 0.001 \\
\hline $\mathrm{LH}^{\mathrm{b}}(\mathrm{mlU} / / \mathrm{mL})$ & $7.53 \pm 4.61$ & $9.12 \pm 7.30$ & $4.23 \pm 1.53$ & NS & $<0.001$ & $<0.001$ \\
\hline$m F G^{b}$ & $3.10 \pm 3.29$ & $3.78 \pm 3.93$ & $0.01 \pm 0.12$ & NS & $<0.001$ & $<0.001$ \\
\hline Acne score ${ }^{b}$ & $0.1667 \pm 0.3791$ & $0.4706 \pm 0.7025$ & $0.4265 \pm 0.6063$ & 0.039 & NS & NS \\
\hline $\begin{array}{l}\text { Menstrual cycle } \\
\text { Shortest }^{\mathrm{b}} \text { (day) }\end{array}$ & $51.52 \pm 35.63$ & $42.87 \pm 23.89$ & $27.70 \pm 2.51$ & NS & $<0.001$ & $<0.001$ \\
\hline $\begin{array}{l}\text { Menstrual cycle } \\
\text { Longtest }^{\mathrm{t}} \text { (day) }\end{array}$ & $108.07 \pm 79.06$ & $72.62 \pm 45.56$ & $31.68 \pm 2.84$ & NS & $<0.001$ & $<0.001$ \\
\hline $\mathrm{AFC}^{\mathrm{b}}$ & $35.32 \pm 12.47$ & $31.15 \pm 9.45$ & $14.49 \pm 3.28$ & NS & $<0.001$ & $<0.001$ \\
\hline
\end{tabular}

Values are mean \pm standard.

$P_{1}$ : Comparison between PCOS with and without NAFLD Groups. $P_{2}$ : Comparison between PCOS with NAFLD and control Groups. $P_{3}$ : Comparison between PCOS without NAFLD and control Groups ${ }^{a}$ Compared by T test or one way ANOVA; ${ }^{b}$ Compared by Nonparametric Test (Mann Whitney U test or Kruskal-Wallis test). 
$<91.7,49$ cases) and group B (MEAN value $\geq 91.7$, 126 cases).

BMI, WC, WHR, SBP, DBP, FIN, FBG, HOMAIR, TG, LDL-C, apoB, ALT, AST, AMH, mFG, menstrual cycles, and AFC were significantly higher in group $\mathrm{A}$ than in group $\mathrm{B}(p<0.05)$. There were no differences in age, $2 \mathrm{hGLU}, \mathrm{CHOL}$, apoA, acne score, and other endocrine parameters between the two groups (Table 2).

Prevalence of endocrine disorders and metabolic disturbances was obviously higher in group A than in group B. The prevalence of oligomenorrhea, PCO, PCOS, IR, IFG, hyperlipidemia, central obesity, EBP, and MS was significantly higher in group A than

\section{RTE}

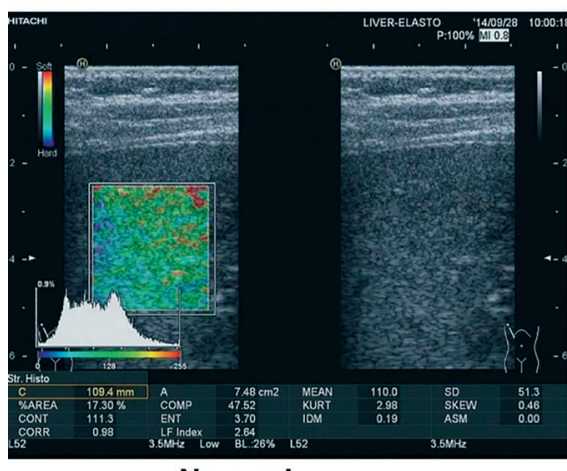

Normal

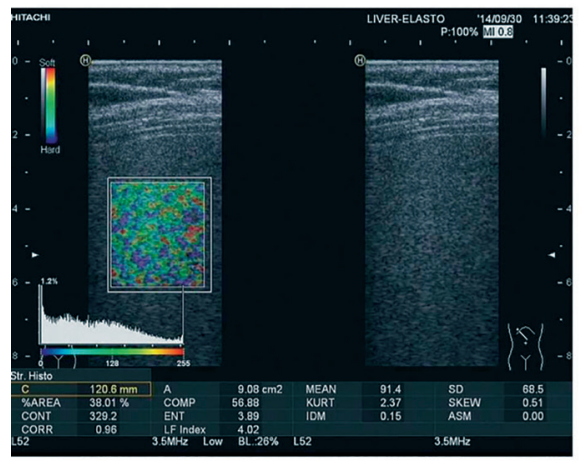

Moderate NAFLD

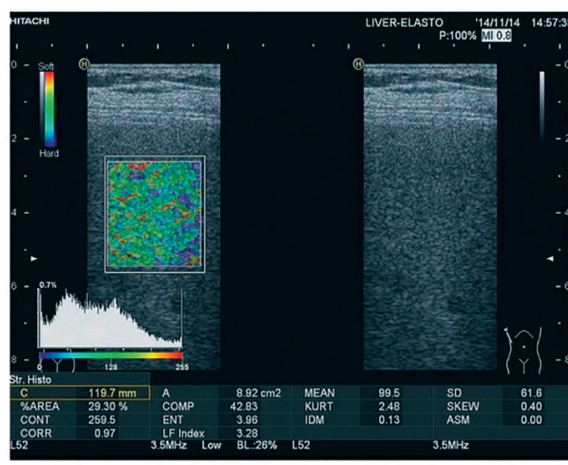

Mild NAFLD

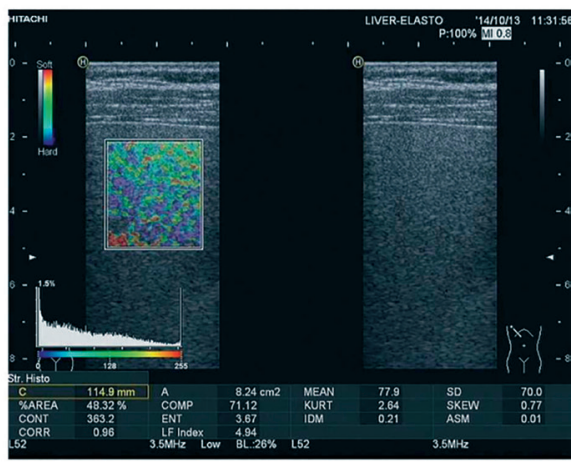

Severe NAFLD

Figure 1. RTE for livers.
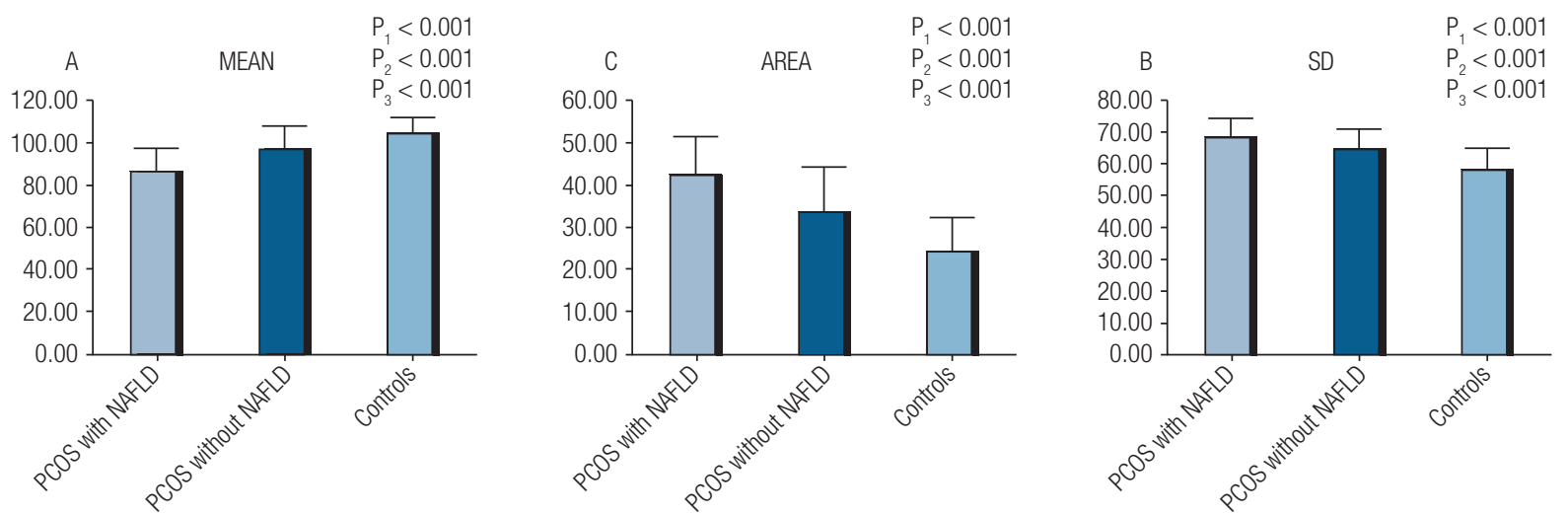

Figure 2. Comparison of RTE parameters between groups PCOS with NAFLD, PCOS without NAFLD, and control. Column bar graphs show significant differences of MEAN, SD, AREA between groups. There were significant trend from PCOS with NAFLD group, PCOS without NAFLD group to control group. P1: Comparison between PCOS with and without NAFLD Groups. P2: Comparison between PCOS with NAFLD and control Groups. P3: Comparison between PCOS Without NAFLD and control Groups. 
Table 2. Comparison of clinical characteristics between the groups formed on the basis of the cutoff value of MEAN

\begin{tabular}{|c|c|c|c|}
\hline & $\begin{array}{c}\text { Group A } \\
\text { (MEAN < 91.7) }\end{array}$ & $\begin{array}{c}\text { Group B } \\
\text { (MEAN } \geq 91.7 \text { ) }\end{array}$ & $p$ \\
\hline $\mathbf{N}$ & 49 & 126 & \\
\hline $\mathrm{Age}^{\mathrm{a}}$ & $29.31 \pm 4.47$ & $28.77 \pm 4.59$ & NS \\
\hline$B M^{a}$ & $24.84 \pm 2.73$ & $21.80 \pm 2.93$ & $<0.001$ \\
\hline$W C^{a}$ & $84.73 \pm 8.70$ & $74.65 \pm 8.35$ & $<0.001$ \\
\hline WHR $^{\mathrm{a}}$ & $0.8683 \pm 0.0644$ & $0.8147 \pm 0.0682$ & $<0.001$ \\
\hline SBPa & $120.90 \pm 10.20$ & $113.68 \pm 11.05$ & $<0.001$ \\
\hline DBPa & $78.73 \pm 7.53$ & $72.28 \pm 9.12$ & $<0.001$ \\
\hline $\mathrm{FBG}^{\mathrm{D}}$ & $5.20 \pm 0.76$ & $4.801 \pm 0.39$ & $<0.001$ \\
\hline $2 h G L U^{b}$ & $7.51 \pm 2.20$ & $7.31 \pm 2.49$ & NS \\
\hline$F \mathbb{N}^{b}$ & $22.66 \pm 13.12$ & $11.26 \pm 6.32$ & $<0.001$ \\
\hline HOMA-IR & $5.36 \pm 4.27$ & $2.43 \pm 1.53$ & $<0.001$ \\
\hline $\mathrm{CHOL}^{\mathrm{a}}$ & $4.83 \pm 1.06$ & $4.70 \pm 0.82$ & NS \\
\hline $\mathrm{TG}^{\mathrm{b}}$ & $1.72 \pm 0.99$ & $1.01 \pm 0.58$ & $<0.001$ \\
\hline HDLCa & $1.30 \pm 0.35$ & $1.47 \pm 0.30$ & 0.002 \\
\hline LDLC $^{\mathrm{a}}$ & $3.07 \pm 0.92$ & $2.73 \pm 0.73$ & 0.017 \\
\hline $\mathrm{apoA}^{\mathrm{a}}$ & $1.43 \pm 0.36$ & $1.457 \pm 0.30$ & NS \\
\hline $\mathrm{apoB}^{\mathrm{a}}$ & $0.84 \pm 0.21$ & $0.75 \pm 0.19$ & 0.013 \\
\hline $\mathrm{ALT}^{\mathrm{b}}$ & $22.904 \pm 14.23$ & $13.04 \pm 5.61$ & $<0.001$ \\
\hline $\mathrm{AST}^{\mathrm{b}}$ & $28.07 \pm 27.04$ & $15.82 \pm 8.79$ & 0.025 \\
\hline $\mathrm{AMH}^{\mathrm{a}}$ & $9.79 \pm 4.82$ & $6.85 \pm 4.53$ & 0.001 \\
\hline$T^{\mathrm{a}}$ & $1.74 \pm 0.62$ & $1.53 \pm 1.00$ & NS \\
\hline$P R L^{a}$ & $13.75 \pm 7.96$ & $15.23 \pm 7.11$ & NS \\
\hline $\mathrm{FSH}^{\mathrm{a}}$ & $6.87 \pm 2.14$ & $7.22 \pm 2.40$ & NS \\
\hline $\mathrm{LH}^{\mathrm{a}}$ & $7.89 \pm 7.42$ & $6.43 \pm 4.78$ & NS \\
\hline$m F G^{a}$ & $3.41 \pm 3.53$ & $1.64 \pm 3.18$ & 0.002 \\
\hline Acne score ${ }^{a}$ & $0.4091 \pm 0.7256$ & $0.3934 \pm 0.6106$ & NS \\
\hline $\begin{array}{l}\text { Menstrual cycle } \\
\text { Shortest }^{\mathrm{b}} \text { (day) }\end{array}$ & $48.93 \pm 26.01$ & $35.69 \pm 22.04$ & $<0.001$ \\
\hline $\begin{array}{l}\text { Menstrual cycle } \\
\text { Longtest }^{\mathrm{D}} \text { (day) }\end{array}$ & $96.86 \pm 69.07$ & $50.12 \pm 37.50$ & $<0.001$ \\
\hline $\mathrm{AFC}^{\mathrm{a}}$ & $31.9 \pm 12.68$ & $21.86 \pm 10.73$ & $<0.001$ \\
\hline
\end{tabular}

Values are mean \pm standard.

${ }^{a}$ Compared by $T$ test or one way ANOVA.

${ }^{\mathrm{b}}$ Compared by Nonparametric Test (Mann Whitney U test or Kruskal-Wallis test).

in group $\mathrm{B}(p<0.05)$. The prevalence of elevated aminotransferase was also higher in group $\mathrm{A}(p<$ $0.05)$. The prevalence of IGT and DM was slightly higher in group A $(p>0.05)$. Nevertheless, there were no differences in the prevalence of HA and hirsutism between the two groups (Table 3 ).

Receiver operating characteristic (ROC) curves were generated to analyze the diagnostic value of MEAN for elevated aminotransferase: the area under the curve was 0.808 (range 0.721-0.895). With the MEAN level of
91.7 as the cutoff value, the sensitivity and specificity were 0.762 and 0.8 respectively, with the Youden index of 0.562 (Figure 3 ).

Table 3. Comparison of frequency of endocrine disorders and metabolic disturbances between the groups formed on the basis of the cutoff value of MEAN

\begin{tabular}{lcccc}
\hline & $\begin{array}{c}\text { Group A } \\
\text { (MEAN < 91.7) }\end{array}$ & $\begin{array}{c}\text { Group B } \\
\text { (MEAN } \geq \mathbf{9 1 . 7 )}\end{array}$ & X2 & P \\
\hline N & $\mathbf{4 9}$ & $\mathbf{1 2 6}$ & & \\
Oligomenorrhea & $44(89.80)$ & $53(42.06)$ & 32.536 & $<0.001$ \\
PCO & $46(93.88)$ & $57(45.24)$ & 32.960 & $<0.001$ \\
bHA & $17(34.69)$ & $40(31.75)$ & 0.140 & NS \\
Hirsutism & $11(22.45)$ & $33(26.19)$ & 0.262 & NS \\
PCOS & $46(93.88)$ & $55(43.65)$ & 36.469 & $<0.001$ \\
IR & $41(83.67)$ & $39(30.95)$ & 39.515 & $<0.001$ \\
IFG & $3(6.12)$ & $1(0.79)$ & 4.485 & $<0.05$ \\
IGT & $11(22.45 \%)$ & $26(20.63)$ & 0.070 & NS \\
DM & $2(4.08)$ & $4(3.17)$ & 0.088 & NS \\
Hyperlipidemia & $19(38.77)$ & $22(17.46)$ & 8.935 & $<0.05$ \\
Central obesity & $39(79.59)$ & $33(26.19)$ & 41.547 & $<0.001$ \\
EBP & $14(28.57)$ & $17(13.49)$ & 5.504 & $<0.05$ \\
MS & $17(34.69)$ & $6(4.76)$ & 27.689 & $<0.001$ \\
Elevated & $9(18.37)$ & $2(1.59)$ & 16.864 & $<0.001$ \\
aminotransferase & & & & \\
\hline
\end{tabular}

Values are number $(\%)$.

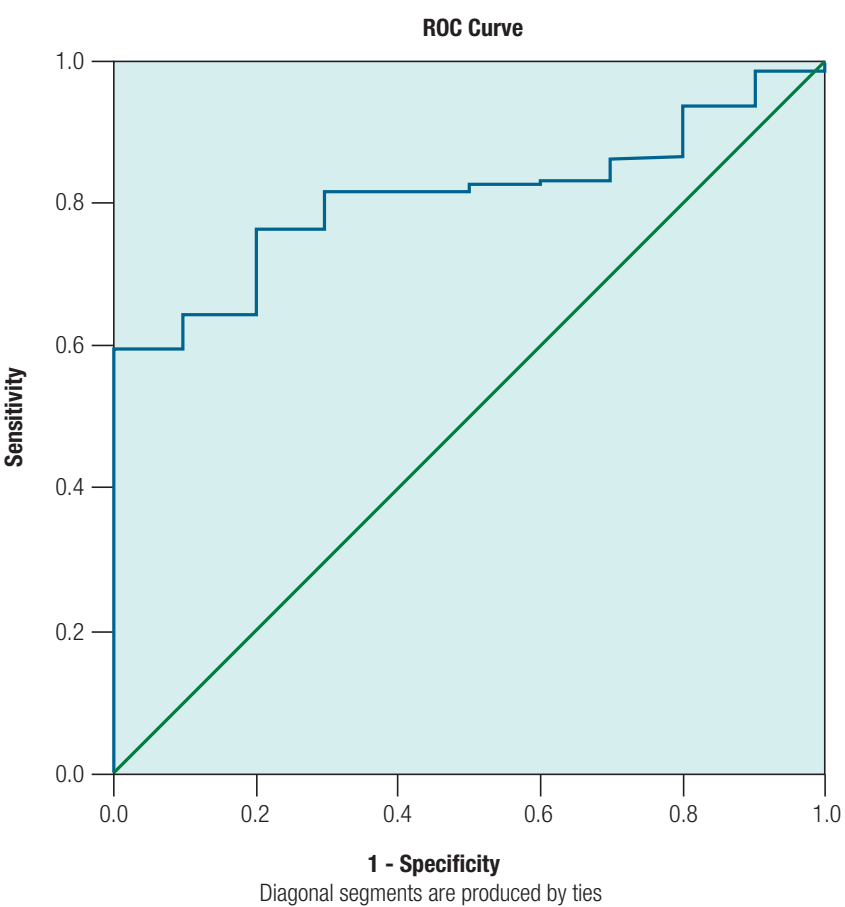

Figure 3. ROC curves for the diagnostic value of MEAN for elevated aminotransferase. ROC curves for MEAN value exhibit reasonable overall performance to discriminate patients with and without elevated aminotransferase. 
In multiple linear regression analyses, the MEAN value was a dependent variable. Independent variables included AGE, BMI, WC, WHR, mFG, FIN, FBP, HOMA-IR, AMH, CHOL, TG, LDL-C, HDL-C, apoA, apoB, ALT, AST, SBP, DBP, MS, and PCOS. The results showed that only PCOS, WC, and MS were stand-alone independent factors associated with the MEAN value among the 140 subjects without NAFLD $(p<0.05)$.

When AREA was a dependent variable (instead of MEAN), PCOS, WC, and MS were also in the list of stand-alone independent factors $(p<0.05)$.

\section{DISCUSSION}

NAFLD is related to obesity, MS, IR, DM, and other metabolic disorders. These metabolic disorders are more frequent among patients with PCOS than among normal individuals. Studies confirmed that patients with PCOS are at a higher risk of NAFLD than controls are $(5,6)$, and this finding is receiving more attention from gynecologists.

RTE is an excellent supplement to conventional ultrasonography. Nonetheless, it is mainly used for diagnosis of focal lesions with impressive accuracy in daily practice. In RTE, elastic strain is a relative indicator of texture stiffness. Compared to other elastographic methods such as transient elastography (TE), the use of RTE with NAFLD has been rarely reported, not to mention the populations with PCOS.

So far, to our knowledge, this is the first study on RTE for assessment of NAFLD in patients with PCOS as compared to healthy controls.

With rapid development of technologies, RTE has been used in more organs than before. The new RTE system has better resolution than the old devices did. Owing to the lower working frequency, the new probe allows for measurements in deeper locations, e.g., in patients with obesity. Automatic displacement of the liver parenchyma is induced by the heartbeat without any pressure from the operator. There is also a sinus curve beneath the RTE image, and this curve shows accuracy of the measurements (16). These technologies reduce interobserver variability remarkably $(28)$.

Semiquantitative parameters of strain histograms of RTE may offer more information about texture elasticity. This approach is reported to be superior to visual scoring for assessment of target strain. In addition, target size has no effect on the strain histogram (29). There is intrinsic relevance among all the 11 semiquantitative strain parameters. Several authors developed different scoring systems based on the parameters calculated with different formulas, and currently there is no consensus $(17,18)$. In this study, we analyzed only the main parameters including MEAN, AREA, and SD, especially MEAN.

Our research confirmed that MEAN decreases in PCOS with NAFLD, while AREA and SD increase as compared to PCOS without NAFLD and healthy controls; these results are in agreement with other studies $(12,16,17,29)$. This finding may be explained theoretically as follows: a softer tissue can be compressed more easily than a stiffer tissue; thus, the displacement of the reflected ultrasound echoes (MEAN) is smaller in the stiffer tissue. AREA is the ratio of low-strain (stiffer) region values. Higher AREA reflects a relatively uneven and harder region of interest. Histologically speaking, major researchers reported that fibrosis associated with hepatic stiffness could be assessed by RTE (30). Furthermore, some researchers believe that steatosis could also affect liver stiffness. Research by Orlacchio and cols. showed that tissue mean elasticity varies consistently with steatosis. Other researchers also found that the steatosis grade is independently associated with a liver elastographic parameter (29,31-33).

In our study, low MEAN was associated with higher WC, BMI, and lipid and transaminase levels as well as with greater endocrine and metabolic disturbances. ROC curve analysis confirmed that MEAN has a good diagnostic value for elevated aminotransferase. These results corroborated the diagnostic value of semiquantitative strain parameters of RTE for NAFLD in addition to conventional ultrasonography.

We found that PCOS patients without NAFLD also had significantly lower MEAN and higher AREA as compared to controls. The levels of MEAN and AREA in PCOS patients without NAFLD were intermediate between those in PCOS patients with NAFLD and healthy controls. According to multiple linear regression analyses, besides WC and MS, PCOS is also a stand-alone independent factor associated with MEAN or AREA values among the subjects without NAFLD. It seems that strain parameters in RTE may distinguish alterations in the liver parenchyma more sensitively: before visual detection by conventional ultrasonic examination. Further research is needed to confirm this notion.

The lack of histological data from livers is a limitation of this study. Nonetheless, the PCOS 
patients of reproductive age came to our department mostly regarding fertility or menstrual problems, and the controls for a health check-up. Therefore, it was inappropriate for them to undergo an invasive liver biopsy. Nevertheless, comparison of MRI and RTE during assessment of liver changes between patients with PCOS and healthy controls may provide more information.

In conclusion, semiquantitative RTE analysis is useful for distinguishing PCOS patients with or without NAFLD and controls. The proposed method may be able to reveal liver parenchyma alterations in patients with PCOS more sensitively. More studies are needed on semiquantitative RTE analysis for diagnosis of NAFLD and other diffuse liver diseases in various populations including patients with PCOS.

Acknowledgment: we gratefully acknowledge the contributions of Hui Zhi, Yabo Yang, Ling Li, Wenchang Yu, Xinhua Ma, Jinglinag Ruan, and Jiayi Wu.

Funding: the study was supported in part by the National Natural Science Foundation of China (grant \# 81402168), the National Natural Science Youth Fund of China (grant \# 81703784), the 5010 Programs Fund for clinical medicine research at Sun Yat-sen University (grant \# 2014005), the Science and Technology research project of Guangzhou city (grant \# 2014Y200512), and the Science and Technology Project of Guangdong Province (grant \# 2018YJ034).

Disclosure: no potential conflict of interest relevant to this article was reported.

\section{REFERENCES}

1. Mathiesen UL, Franzen LE, Aselius H, Resjo M, Jacobsson L, Foberg $U$, et al. Status of homocysteine in polycystic ovary syndrome (PCOS). J Clin Diagn Res. 2014;8(2):31-3.

2. Fung J, Lee CK, Chan M, Seto WK, Lai CL, Yuen MF. High prevalence of non-alcoholic fatty liver disease in the Chinese-results from the Hong Kong liver health census. Liver Int. 2015;35(2):542-9.

3. Jayasena CN, Franks S. The management of patients with polycystic ovary syndrome. Nat Rev Endocrinol. 2014;10(10):624-36.

4. Fauser BC, Tarlatzis BC, Rebar RW, Legro RS, Balen AH, Lobo R, et al. Consensus on women's health aspects of polycystic ovary syndrome (PCOS): the Amsterdam ESHRE/ASRM-Sponsored 3rd PCOS Consensus Workshop Group. Fertil Steril. 2012;97(1):28-38.e25.

5. Ramezani-Binabaj M, Motalebi M, Karimi-Sari H, Rezaee-Zavareh MS, Alavian SM. Are women with polycystic ovarian syndrome at a high risk of non-alcoholic Fatty liver disease; a meta-analysis. Hepat Mon. 2014;14(11):e23235.

6. Romanowski MD, Parolin MB, Freitas AC, Piazza MJ, Basso J, Urbanetz AA. Prevalence of non-alcoholic fatty liver disease in women with polycystic ovary syndrome and its correlation with metabolic syndrome. Arq Gastroenterol. 2015;52(2):117-23.

7. Targher G, Rossini M, Lonardo A. Evidence that non-alcoholic fatty liver disease and polycystic ovary syndrome are associated by necessity rather than chance: a novel hepato-ovarian axis? Endocrine. 2016;51(2):211-21.

8. Ferraioli G, Filice C, Castera L, Choi B I, Sporea I, Wilson SR, et al. WFUMB guidelines and recommendations for clinical use of ultrasound elastography: Part 3: liver. Ultrasound Med Bio. 2015;41(5):1161-79.

9. Papagianni M, Sofogianni A, Tziomalos K. Non-invasive methods for the diagnosis of nonalcoholic fatty liver disease. World J Hepatol. 2015;7(4):638-48.

10. Kim YW, Kwon JH, Jang JW, Kim MJ, Oh BS, Chung KW, et al. Diagnostic usefulness of real-time elastography for liver fibrosis in chronic viral hepatitis B and C. Gastroenterol Res Pract. 2014;2014:210407.

11. Colombo S, Buonocore M, Del PA, Jamoletti C, Elia S, Mattiello $M$, et al. Head-to-head comparison of transient elastography (TE), real-time tissue elastography (RTE), and acoustic radiation force impulse (ARFI) imaging in the diagnosis of liver fibrosis. J Gastroenterol. 2012;47(4):461-9.

12. Morikawa H, Fukuda K, Kobayashi S, Fujii H, Iwai S, Enomoto M, et al. Real-time tissue elastography as a tool for the noninvasive assessment of liver stiffness in patients with chronic hepatitis $\mathrm{C}$. J Gastroenterol. 2011;46(3):350-8.

13. Ferraioli G, Tinelli C, Malfitano A, Dal Bello B, Filice G, Filice C, et al. Performance of real-time strain elastography, transient elastography, and aspartate-to-platelet ratio index in the assessment of fibrosis in chronic hepatitis C. AJR Am J Roentgenol. 2012;199(1):19-25.

14. Fujimoto K, Kato M, Kudo M, Yada N, Shiina T, Ueshima K, et al. Novel image analysis method using ultrasound elastography for noninvasive evaluation of hepatic fibrosis in patients with chronic hepatitis C. Oncology. 2013;84 Suppl 1:3-12.

15. Yada N, Kudo M, Morikawa H, Fujimoto K, Kato M, Kawada N. Assessment of liver fibrosis with real-time tissue elastography in chronic viral hepatitis. Oncology. 2013;84 Suppl 1:13-20.

16. Schenk JP, Alzen G, Klingmuller V, Teufel U, EI SS, et al. Measurement of real-time tissue elastography in a phantom model and comparison with transient elastography in pediatric patients with liver diseases. Diagn Interv Radio. 2014;20(1):90-9.

17. Ge L, Shi B, Song YE, LiY, Wang S, Wang X. Clinical value of realtime elastography quantitative parameters in evaluating the stage of liver fibrosis and cirrhosis. ExpTher Med. 2015;10(3):983-90.

18. Wang J, Guo L, Shi X, Pan W, BaiY, Ai H. Real-time elastography with a novel quantitative technology for assessment of liver fibrosis in chronic hepatitis B. Eur J Radiol. 2012;81(1):31-6.

19. Revised 2003 consensus on diagnostic criteria and long-term health risks related to polycystic ovary syndrome (PCOS). Hum Reprod. 2004;19(1):41-7.

20. Mathiesen UL, Franzen LE, Aselius H, Resjo M, Jacobsson L, Foberg $U$, et al. Increased liver echogenicity at ultrasound examination reflects degree of steatosis but not of fibrosis in asymptomatic patients with mild/moderate abnormalities of liver transaminases. Dig Liver Dis. 2002;34(7):516-22.

21. Zhao X, He Z, Mo Y, Chen X, Chen Y, Yang D. Determining the normal cut-off levels for hyperandrogenemia in Chinese women of reproductive age. Eur J Obstet Gynecol Reprod Biol. 2011;154(2):187-91.

22. Zhao X, Ni R, Li L, MoY, Huang J, Huang M, et al. Defining hirsutism in Chinese women: a cross-sectional study. Fertil Steril. 2011;96(3):792-6.

23. Pochi PE, Shalita AR, Strauss JS, Webster SB, Cunliffe WJ, Katz HI, et al. Report of the Consensus Conference on Acne Classification. J Am Acad Dermatol. 1991;24(3):495-500.

24. Chen X, Yang D, Li L, Feng S, Wang L. Abnormal glucose tolerance in Chinese women with polycystic ovary syndrome. Hum Reprod. 2006;21(8):2027-32. 
25. Chen X, Yang D, Li L, Feng S, Wang L. Appropriate BMI levels for PCOS patients in Southern China. Hum Reprod. 2010;25(5):1295-302.

26. WHO. Definition and diagnosis of diabetes mellitus and intermediate hyperglycaemia Report of aWHO/IDF consultation. Available at: http:// www.who.int/diabetes/publications/diagnosis_diabetes2006/en.

27. Rezaianzadeh A, Namayandeh SM, Sadr SM. National Cholesterol Education Program Adult Treatment Panel III Versus International Diabetic Federation Definition of Metabolic Syndrome, Which One is Associated with Diabetes Mellitus and Coronary Artery Disease? Int J Prev Med. 2012;3(8):552-8.

28. Carlsen JF, Ewertsen C, Saftoiu A, Lonn L, Nielsen MB. Accuracy of visual scoring and semi-quantification of ultrasound strain elastography - a phantom study. PLoS One. 2014;9(2):e88699.

29. Orlacchio A, Bolacchi F, Antonicoli M, Coco I, Costanzo E, Tosti D, et al. Liver elasticity in NASH patients evaluated with real-time elastography (RTE). Ultrasound Med Biol. 2012;38(4):537-44.
30. Ochi H, Hirooka M, Koizumi Y, Miyake T, Tokumoto Y, Soga Y, et al. Real-time tissue elastography for evaluation of hepatic fibrosis and portal hypertension in nonalcoholic fatty liver diseases. Hepatology. 2012;56(4): 1271-1278.

31. Yilmaz Y, Yesil A, Gerin F, Ergelen R, Akin H, Celikel CA, et al. Detection of hepatic steatosis using the controlled attenuation parameter: a comparative study with liver biopsy. Scand J Gastroenterol. 2014;49(5):611-6.

32. Chon Y E, Jung KS, Kim SU, Park JY, Park YN, Kim DY, et al. Controlled attenuation parameter (CAP) for detection of hepatic steatosis in patients with chronic liver diseases: a prospective study of a native Korean population. Liver Int. 2014;34(1):102-9.

33. Shen F, Zheng RD, MiYQ, Wang XY, Pan Q, Chen GY, et al. Controlled attenuation parameter for non-invasive assessment of hepatic steatosis in Chinese patients. World J Gastroenterol. 2014;20(16):4702-11. 